\title{
Segmentation of Dermoscopic Images
}

\author{
Komal Lawand \\ (Department of Instrumentation Engineering, R.A.I.T, Navi Mumbai)
}

\begin{abstract}
This paper introduces a new approach for the segmentation of skin lesions in dermoscopic images based on wavelet network (WN). The WN presented here is a member of fixed-grid WNs that is formed with no need of training. R, G, and B values of a dermoscopy image are considered as the network inputs and the network structure formation. Then, the image is segmented and the skin lesions exact boundary is determined accordingly. The segmentation algorithm were applied to 30 dermoscopic images and evaluated with 11 different metrics, using the segmentation result obtained by a skilled pathologist as the ground truth. Experimental results show that this method acts more effectively in comparison with some modern techniques that have been successfully used in many medical imaging problems.
\end{abstract}

Keywords: - - Dermoscopy, image segmentation, melanoma diagnosis, wavelet network (WN).

\section{INTRODUCTION}

Malignant melanoma is the most frequent type of skin cancer and its incidence has been rapidly increasing over the last few decades. According to a WHO report, about 48,000 melanoma related deaths occur worldwide per year. Nevertheless, it is also the most treatable kind of skin cancer, if diagnosed at an early stage. The clinical diagnosis of melanoma is commonly based on the ABCD rule, an analysis of four parameters (asymmetry, border irregularity, color, and dimension), or the 7-points checklist [1] which is a scoring method for a set of different characteristics depending on color, shape, and texture. Dermoscopy is a non-invasive diagnosis technique for the in vivo observation of pigmented skin lesions used in dermatology. Dermoscopic images have great potential in the early diagnosis of malignant melanoma, but their interpretation is time consuming and subjective, even for trained dermatologist's . Therefore, there is currently a great interest in the development of computer-aided diagnosis systems that can assist the clinical evaluation of dermatologists. The standard approach in automatic dermoscopic image analysis has usually three stages: 1) image segmentation; 2) feature extraction and feature selection; and 3) lesion classification.

The segmentation stage is one of the most important since it affects the accuracy of the subsequent steps. However, segmentation is difficult because of the great variety of lesion shapes, sizes, and colors along with different skin types and textures. To address this problem, several algorithms have been proposed. They can be broadly classified as thresholding, edge based or region-based methods. An example of thresholding where a fusion of global thresholding, adaptive thresholding, and clustering is used. Thresholding methods achieve good results when there is good contrast between the lesion and the skin, thus the corresponding image histogram is bimodal, but usually fails when the modes from the two regions overlap. Edge-based methods like the gradient vector flow (GVF), Edge-based approaches perform poorly when the boundaries are not well defined, and for instance when the transition between skin and lesion is smooth. In these situations, the edges have gaps and the contour may leak through them. Another difficulty is the presence of spurious edge points that do not belong to the lesion boundary. They are the result of artifacts such as hair, specular reflections or even irregularities in the skin texture and they may stop the contour preventing it to converge to the lesion boundary. Region-based approaches have also been used. Region-based approaches have difficulties when the lesion or the skin region are textured or have different colors present, which leads to over segmentation [2]. Whereas WN takes full advantage of the characteristics of WT in denoising, background reduction, and recovery of the characteristic information provide the frequency information and temporal information.

\section{WAVELET NETWORK}

Wavelet transform is one of a best tool for determine the location of low frequency area and high frequency area. WNs are divided into two groups: Adaptive wavelet networks (AWNs) and fixed-grid wavelet networks (FGWNs) [3].

Adaptive wavelet network is continuous wavelet transform whereas FGWN is discrete wavelet transform. Due to numerous shortcomings of AWNs like for example, complex calculations, sensitivity to initial values, and the problem of measuring initial values, their application is limited. In an FGWN, the outer parameters of the network like number of wavelets, scale, and shift parameters value are determined beforehand, and only inner parameters of the network which is weights are specified by algorithms similar to the least 
squares. In fact such networks do not need training. In AWNs, initial values of network parameters including weights of neurons, shifts, and scales of wavelets are selected randomly or using other methods. These parameters are then updated in the training stage by means of techniques such as gradient descent or back propagation (BP). Then, the optimized values of network parameters are calculated. Whereas contrary to AWNs, in an FGWN, the number of wavelets, as well as the scale and shift parameters, can be determined in advance and the only unknown parameters are the weight coefficients which are calculated through methods such as least squares. So in proposed FGWN, there is no need to specify random initial values for parameters or to use gradient descent, BP, or other iterative methods. Normally, in training stage of an adaptive network, all the parameters change; on contrary, in FGWN only, the weights are specified during a non iterative process. Thus, it could be concluded that FGWNs do not need training procedure [3].

2.1 Structure of wavelet network

The output signal of a WN with one output $\mathrm{y}, \mathrm{d}$ inputs $\mathrm{X}=(\mathrm{X} 1, \mathrm{X} 2 \ldots \mathrm{X} \mathrm{d})^{\mathrm{T}}$ and $\mathrm{q}$ wavelet neuron in the hidden layer is

$$
y=\sum_{i=1}^{q} w i \Psi_{m i}, n i(x)
$$

Where wi, i=1, 2, ...q are weight coefficients $\Psi_{\mathrm{mi} \text {, ni }}$ are dilated and translated version of the mother wavelet function $\Psi$. And mi and ni are scale and shift parameters respectively.

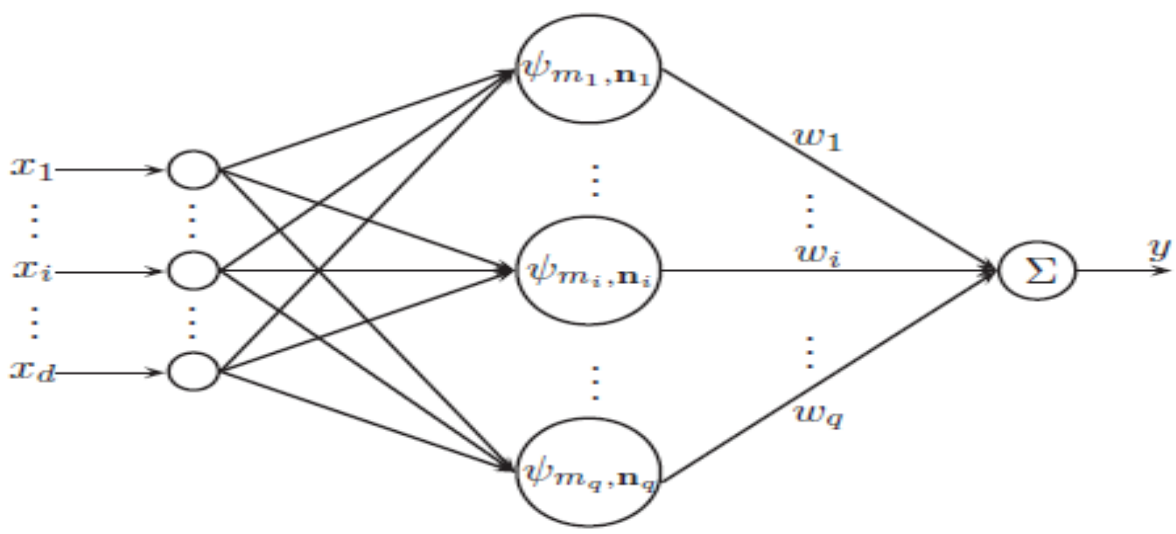

Fig 1: Three layered WN structure with one hidden layer.

\section{SEGMENTATION ALGORITHM}

From images database, a number of images are randomly chosen for formation of FGWN. At first the values of $\mathrm{R}, \mathrm{G}$, and $\mathrm{B}$ matrices of each color dermoscopic image are mapped into $[0,1]$ range by performing normalization process.

$$
\mathrm{Xq}(\mathrm{k}) \text { new }=\frac{\mathrm{Xq}(\mathrm{k}) \text { old }-\mathrm{tk}}{T_{k}-\mathrm{tk}^{k}}
$$

$\mathrm{Xq}(\mathrm{k})$ new is the value of each color matrix after normalization (located in $[0,1]$ range), and tk and Tk are minimum and maximum values of these matrices, respectively. Then, an FGWN is formed with three inputs, a hidden layer, and an output. In order to form the FGWN, the values of three color matrices are considered as network inputs. These matrices are related to the five chosen images from the selected images for segmentation. From these images, some pixels are selected randomly ranging from 1000 to 5000 pixels for a $485 \times 716$ image. If the pixel is inside the lesion, network output will be considered as 0 , and if the pixel is outside the lesion, the output will be considered as 1 .

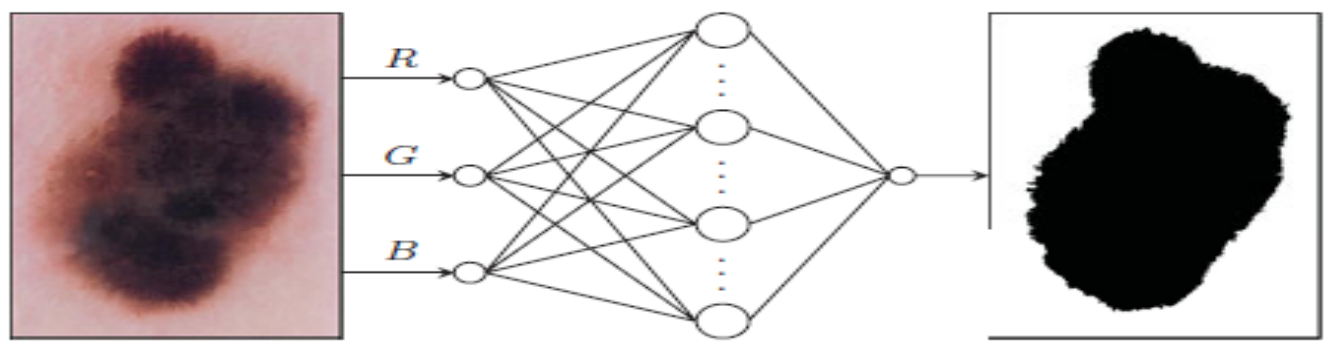

Fig2 : FGWN for segmentation of dermoscopic images. 
In this way, the FGWN is formed. After that, the value of the three matrices R, G, and B for each pixel are considered as FGWN inputs, and the output of FGWN is a binary image that shows the segmented of original image. This study employs a collection of dermoscopic images taken from a specialized skin laboratory as its database. This database includes 1039 dermoscopic images from different parts of the body taken under the same environmental conditions. All of these images are 24-bit RGB color, with $485 \times 716$ size, and are taken from patients suspected to melanoma. Whether or not the patients have caught the disease is determined by biopsy and is diagnosed by the dermatologist. A large number of database images are very similar. Accordingly, when the images were perused and segmented by a skilled dermatologist (Dr. M. Mokhtari, a skilled specialist with over ten years of experience in dermoscopic image analysis), they were naturally categorized to 30 distinct groups. In fact, each of our selected 30 images is a representative of a group. From each group, one image was randomly chosen, and for each image, a manual segmentation was performed in fullsized printed images. These images were scanned with the same resolution as the primary images and used as ground truth of automatic segmentation.

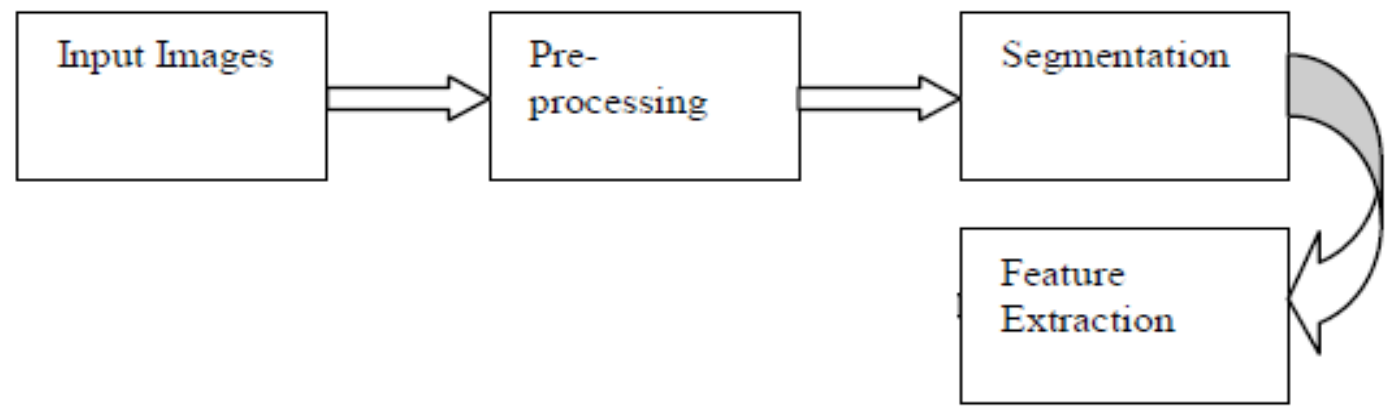

Fig3: Basic block diagram for segmentation

The pre processing step removes the undesirable parts, enhances the image, corrects the image skew and removes noise from the image. Noise is one of the problems of the melanoma and benign images. Among different filters, the mean filter was chosen because of its efficiency. A kind of noise that might appear on dermoscopic image during transmission, storage, and processing is impulse noise. The researchers have cancelled this noise based on the combination of FGWN and median filter.

An example of the segmentation results obtained by the various methods is given in Fig. 3 which shows one of the ground truth segmentation together with the results by all five other methods. The algorithms that compared with the FGWN are AT [2], GVF [4], FBSM [5], and NN [6].
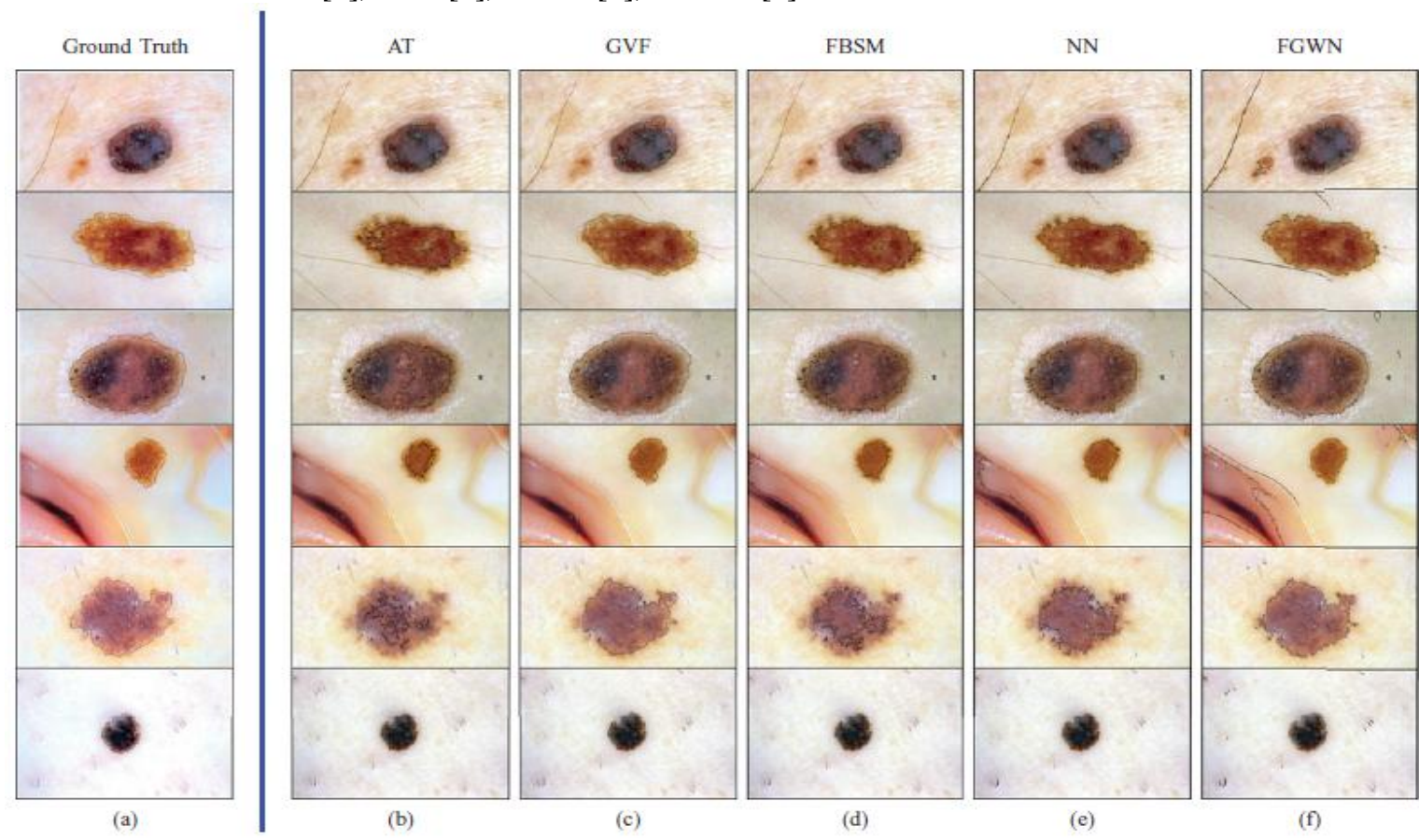

Fig4: Segmentation results. (a) GT results, (b) AT results, (c) GVF results, (d) FBSM results, (e) NN results, and (f) FGWN results [6]. 
The fig4 shows AT achieves good results when there is a good contrast between the lesion and the skin. The GVF is a well-known technique frequently used in many medical imaging. In FBSM, dermoscopic image segmentation is based on fuzzy methods, which yields fairly good results. Since the central idea of WNs has its roots in NNs; an NN is also used for segmentation. Among the 30 images selected for segmentation using the FGWN, five images were used for building the network structure formation of the wavelet lattice, Referring to Figure, it can be observed that the segmentation produced by the proposed method FGWN is smoother than those by four other algorithms. Clearly, smoother border is more realistic and also conforms better than the manual segmentation derived by the specialist [6].

After segmentation post processing is done. Since extracting the features of lesion is the most essential part of diagnosing melanoma, extracting the exact boundary of lesion is a vital task. For this, after segmentation with an FGWN the space between two shapes is filled, extra parts are eliminated, and the noise is removed. Then, the exact boundary of lesion is extracted. This is done by appropriate morphological processes, including erosion, dilation, closing and opening, and region filling. Size, shape, and kind of structuring elements were based on images dimensions and type of their objects that are selected tentatively and provisionally.

As mentioned before, segmentation is the most important and critical stage of the three stages of automatic diagnosis of melanoma which has a very significant role in the final outcome. Because of this reason, the performance of this state should be examined by means of appropriate criteria. In this study, 11 criteria of standard evaluation are employed which have been used in a great number of related research. To define these metrics, let GT and SA denote the ground truth segmentation obtained by the medical expert and results of a segmentation algorithm, respectively. Both GT and SA are binary images and all the pixels inside the curve have label 1 and all the others have label 0 .

Segmentation Performance on the 30 Images From Database

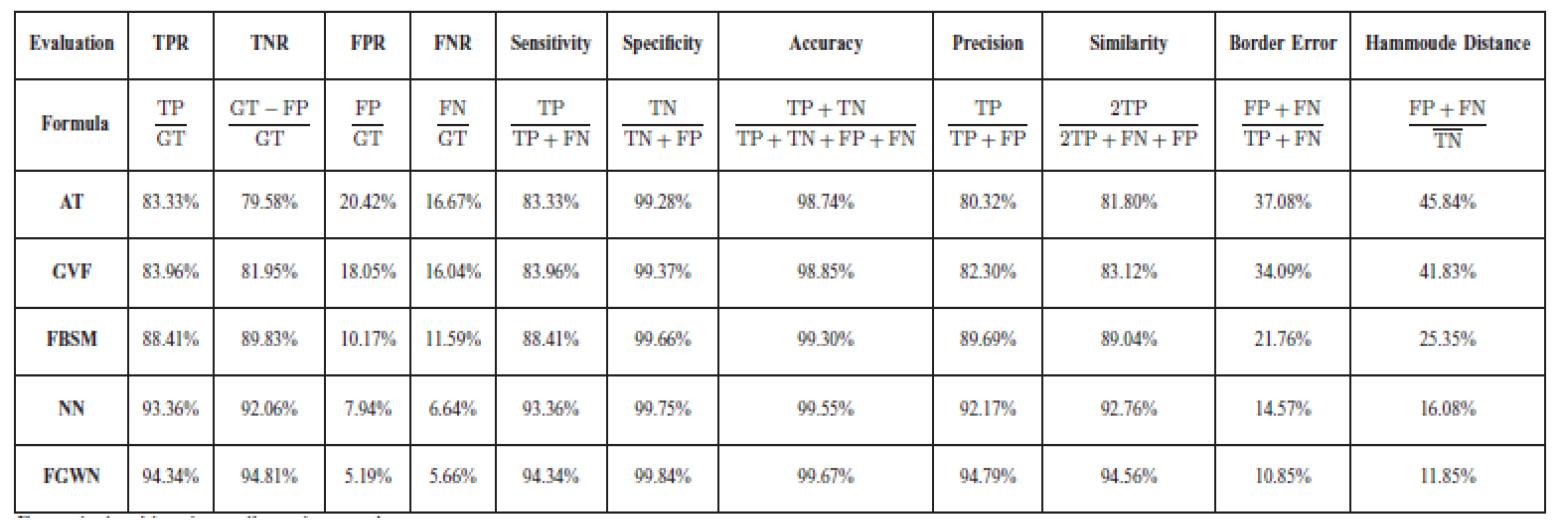

Fig5: Segmentation performance on the 30 images from database [6].

TP: true positive, lesion pixels correctly classified as lesion.

FP: false positive, skin pixels incorrectly identified as lesion.

TN: true negative, skin pixels correctly identified as skin.

FN: false negative, lesion pixels incorrectly identified as skin.

As depicted in this table, the values of proposed algorithm for all the evaluation criteria are better than other methods. In a similar vein, it is evident from this table that the FGWN algorithm has an appropriate level of specificity (99.84\%) and sensitivity (94.34\%). This means that the FGWN algorithm diagnoses the lesion boundary properly therefore, the lesion boundary that is the most significant feature in detecting melanoma is extracted by FGWN with an acceptable accuracy (99.67\%) and precision (94.79\%). Method also achieved to measure correct TPR and TNR (94.31\%and $94.81 \%$ respectively) with border error of only $10.85 \%$. FGWN method is quite simple and considering the satisfactory results of this study.

\section{CONCLUSION}

We have seen that in this study, a new approach is proposed for segmentation of dermoscopic images based on FGWN. The R, G, and B values of a dermoscopy image are considered as FGWN inputs and the OLS algorithm is used to determine the network weights and to optimize the network structure. The proposed method with four methods (AT, GVF, FBSM, and NN) and hand segmentation by a specialist and based on 11 criteria showed better results in comparison to similar previous studies. The developed algorithm hence provides a useful tool as a first stage in the automatic or semiautomatic analysis of skin lesion images. 


\section{REFERENCES}

[1] A. G. Isasi, B. G. Zapirain, and A. M. Zorrilla, "Melanomas non-invasive diagnosis application based on the ABCD rule and pattern recognition image processing algorithms," J. Comput. Biol. Med., vol. 41, no. 9, pp. 742-755, Sep. 2011.

[2] M. Silveria, J. C. Nascimento, J. S. Marques, A. R. S. MarcalT. Mendonca, S. Yamauchi, J. Maeda, and J. Rozeira, "Comparison of segmentation methods for melanoma diagnosis in dermoscopy images,"IEEE Trans. Signal Process. vol. 3, no. 1, pp. 35-45, Mar. 2009.

[3] K.-S. Cheng, J.-S. Lin, and C.-W. Mao, "Techniques and comparative analysis of neural network systems and fuzzy systems in medical image segmentation," Fuzzy Theor. Syst. Tech. Appl., vol. 3, pp. 973-1008, 1999.

[4] B. Erkol, R. H.Moss, R. J. Stanley,W. V. Stoecker, and E. Hva-tum, "Automatic lesion boundary detection in dermoscopy images using gradientvector flow snakes," Skin Res. Technol., vol. 11, no. 1, pp. 17-26, Feb.2005.

[5] J. Maeda, A. Kawano, S. Saga, and Y. Suzuki, "Unsupervised perceptual segmentation of natural color images using fuzzy-based hierarchical algorithm," in Proc. 15th Scandinavian Conf. Image Analysis, 2007,pp. 642-671.

[6] Amir Reza Sadri, Maryam Zekri*, Member, IEEE, Saeed Sadri, Niloofar Gheissari, Mojgan Mokhtari,and Farzaneh Kolahdouzan,"Segmentation of Dermoscopy Images UsingWavelet Networks", IEEE transactions on biomedical engineering, vol. 60, no. 4, april 2013. 